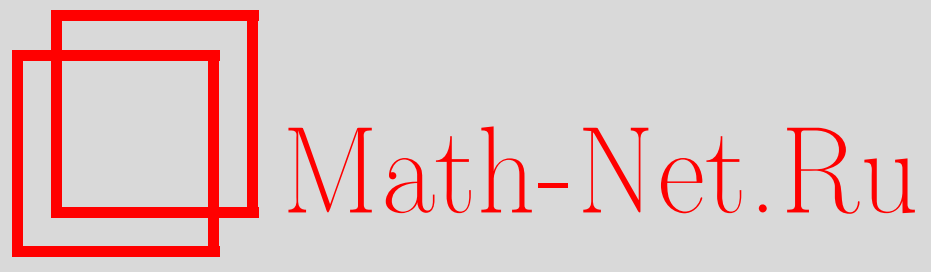

Г. Г. Ониани, О взаимосвязи между сходимостями по прямоугольникам и по сферам кратных рядов Хаара, УМH, 2012, том 67, выпуск 1, 185-186

DOI: https://doi.org/10.4213/rm9464

Использование Общероссийского математического портала Math-Net.Ru подразумевает, что вы прочитали и согласны с пользовательским соглашением http://www . mathnet.ru/rus/agreement

Параметры загрузки:

IP: 18.208 .226 .222

26 апреля 2023 г., 16:36:41

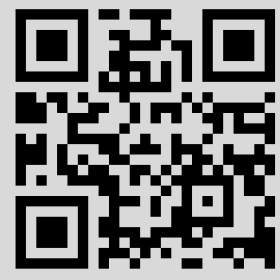




\section{О взаимосвязи между сходимостями по прямоугольникам и по сферам кратных рядов Хаара}

\section{Г. Г. Ониани}

Пусть $n \geqslant 2, \mathbb{I}^{n}=[0,1]^{n}$, и пусть $\left\{h_{k}\right\}_{k \in \mathbb{N}^{n}}$ есть $n$-кратная система Хаара. Для $n$-кратного ряда Хаара

$$
\sigma=\sum_{k \in \mathbb{N}^{n}} c_{k} h_{k}
$$

и для $x \in \mathbb{I}^{n}, m \in \mathbb{N}^{n}$ и $r>0$ через $S_{m}(\sigma)(x), S_{r}(\sigma)(x), G_{m}(\sigma)(x)$ и $G_{r}(\sigma)(x)$ обозначим прямоугольную частичную сумму, сферическую частичную сумму, прямоугольный общий член и сферический общий член соответственно, т. е.

$$
\begin{aligned}
S_{m}(\sigma)(x) & =\sum_{k_{1}=1}^{m_{1}} \cdots \sum_{k_{n}=1}^{m_{n}} c_{k} h_{k}(x), & S_{r}(\sigma)(x) & =\sum_{k_{1}^{2}+\cdots+k_{n}^{2} \leqslant r^{2}} c_{k} h_{k}(x), \\
G_{m}(\sigma)(x) & =c_{m} h_{m}(x), & G_{r}(\sigma)(x) & =\sum_{k_{1}^{2}+\cdots+k_{n}^{2}=r^{2}} c_{k} h_{k}(x),
\end{aligned}
$$

где $m=\left(m_{1}, \ldots, m_{n}\right), k=\left(k_{1}, \ldots, k_{n}\right)$ и сумма по пустому множеству индексов считается равной 0. Если $\sigma=\sigma(f)$ - ряд Фурье-Хаара функции $f \in L\left(\mathbb{I}^{n}\right)$, то в вышеприведенных обозначениях вместо $\sigma$ будем использовать $f$.

Если $n \geqslant 2$ и $1 \leqslant \ell \leqslant n-1$, то $\ell$-мерным сечением последовательности $\left(a_{m}\right)_{m \in \mathbb{N}^{n}}$ будем называть каждую последовательность, получаемую из $\left(a_{m}\right)_{m \in \mathbb{N}^{n}}$ фиксированием некоторых $n-\ell$ среди $n$ координат индекса $m=\left(m_{1}, \ldots, m_{n}\right)$.

Под сходимостью (сильной сходимостью) последовательности $\left(a_{m}\right)_{m \in \mathbb{N}^{n}}$ мы понимаем сходимость $a_{m}$ к пределу при $\min \left\{m_{1}, \ldots, m_{n}\right\} \rightarrow \infty\left(\right.$ при $\left.\max \left\{m_{1}, \ldots, m_{n}\right\} \rightarrow \infty\right)$.

Заметим, что для последовательности $\left(a_{m}\right)_{m \in \mathbb{N}^{n}}$ следующие условия эквивалентны: 1) $\left(a_{m}\right)_{m \in \mathbb{N}^{n}}$ сильно сходится к $\left.a ; 2\right)\left(a_{m}\right)_{m \in \mathbb{N}^{n}}$ сходится к $a$ и каждое $(n-1)$-мерное сечение $\left(a_{m}\right)_{m \in \mathbb{N}^{n}}$ сильно сходится к $a$.

Известно, что $L\left(\ln ^{+} L\right)^{n-1}\left(\mathbb{I}^{n}\right)$ при каждом $n \geqslant 2$ является наиболее широким интегральным классом, в котором обеспечена сходимость почти всюду рядов Фурье-Хаара как для прямоугольных частичных сумм (см. [1]), так и для сферических частичных сумм (см. [2], [3]). Здесь же заметим, что кубические частичные суммы ряда Фурье-Хаара каждой суммируемой функции (т. е. $S_{m}(f)(x)$ с $m_{1}=m_{2}=\cdots=m_{n}$ ) сходятся почти всюду к $f$ (детали см., например, в [1]).

Наша цель - изучить взаимосвязь между прямоугольной и сферической сходимостями кратных рядов Хаара. Известна следующая теорема Кемхадзе [2].

Tеорема А. Пусть $f \in L\left(\mathbb{I}^{2}\right)$. Если $\lim _{m \rightarrow \infty} S_{m}(f)(x)=f(x)$ для почти всех $x \in \mathbb{I}^{2}$, mo $\lim _{r \rightarrow \infty} S_{r}(f)(x)=f(x)$ для почти всех $x \in \mathbb{I}^{2}$.

Справедливы следующие две теоремы.

Теорема 1. Пусть $n \geqslant 2$ и $\sigma$ есть $n$-кратный ряд Хаара. Если $\sigma$ сходится по прямогольникам в двоично-иррачиональной точке $x$ и прямоугольный общий член $\left(G_{m}(\sigma)(x)\right)_{m \in \mathbb{N}^{n}}$ силъно сходится $\kappa$ 0, то $\sigma$ по сферам сходится в точке $x \kappa$ $\lim _{m \rightarrow \infty} S_{m}(\sigma)(x)$.

Tеорема 2. Пусть $n \geqslant 2 u f \in L\left(\ln ^{+} L\right)^{n-2}\left(\mathbb{I}^{n}\right)$. Тогда для почти всех $x \in \mathbb{I}^{n}$ каждое $(n-1)$-мерное сечение последовательности $\left(G_{m}(f)(x)\right)_{m \in \mathbb{N}^{n}}$ сильно сходит$c \Omega \kappa 0$. 
Из теорем 1 и 2 вытекает следующее обобщение теоремы А.

СледствиЕ 1. Пусть $n \geqslant 2, f \in L\left(\ln ^{+} L\right)^{n-2}\left(\mathbb{I}^{n}\right)$ u $E$ есть множество всех точек $x$, в которых $\lim _{m \rightarrow \infty} S_{m}(f)(x)=f(x)$. Тогда $\lim _{r \rightarrow \infty} S_{r}(f)(x)=f(x)$ для почти всех $x \in E$.

Из следствия 1, принимая во внимание сходимость почти всюду по прямоугольникам рядов Фурье-Хаара функции из $L\left(\ln ^{+} L\right)^{n-1}\left(\mathbb{I}^{n}\right)$, выводим следующий результат Кемхадзе [2].

Следствие 2. Пусть $n \geqslant 2 u f \in L\left(\ln ^{+} L\right)^{n-1}\left(\mathbb{I}^{n}\right)$. Тогда $\lim _{r \rightarrow \infty} S_{r}(f)(x)=f(x)$ для почти всех $x \in \mathbb{I}^{n}$.

Следующая теорема, в частности, показывает, что при $n \geqslant 3$ связь между сходимостью по прямоугольникам и сходимостью по сферам, о которой говорится в следствии 1 , нарушается при расширении интегрального класса $L\left(\ln ^{+} L\right)^{n-2}\left(\mathbb{I}^{n}\right)$.

Теорема 3. Для каждого $n \geqslant 3$ и каждой функции $f \in L \backslash L\left(\ln ^{+} L\right)^{n-2}\left(\mathbb{I}^{n}\right)$ существует равноизмеримая с $f$ функиия $g$ на $\mathbb{I}^{n}$ такая, что

$$
\lim _{m \rightarrow \infty} S_{m}(g)(x)=g(x) \quad \text { u } \quad \limsup _{r \rightarrow \infty}\left|G_{r}(g)(x)\right|=\infty \quad \text { для почти всех } \quad x \in \mathbb{I}^{n} .
$$

\section{Список литературы}

[1] Т.Ш. Зерекидзе, "Сходимость кратных рядов Фурье-Хаара и сильная дифференцируемость интегралов”, Труды Тбилис. матем. ин-та им. А.М. Размадзе АН ГрССР, 76 (1985), 80-99. [2] Г. Г. Кемхадзе, "О сходимости шаровых частичных сумм кратных рядов Фурье-Хаара", Труды Тбилис. матем. ин-та им. А.М. Размадзе АН ГрССР, 55 (1977), 27-38. [3] Г. Г. Ониани, "О расходимости кратных рядов Фурье-Хаара", Докл. РАН, 419:2 (2008), 169-170; англ. пер.: G. G. Oniani, "On the divergence of multiple Fourier-Haar series", Dokl. Math., 77:2 (2008), 203-204.

\section{Г. Г. Ониани (G. G. Oniani)}

Государственный университет им. Акакия Церетели, Кутаиси, Грузия

E-mail: oniani@atsu.edu.ge
Представлено А. Г. Сергеевым Принято редколлегией

11.01 .2012 